\title{
RECONSTRUCTION ANALYSIS-BASED ALGORITHM TO DECOMPOSE A COMPLEX SYSTEM INTO SUBSYSTEMS
}

\author{
JOSEP M. MIRATS TUR ${ }^{\mathrm{a}, *}$, FRANÇOIS E. CELLIER ${ }^{\mathrm{b}, \dagger}$ and RAFAEL M. HUBER ${ }^{\mathrm{a}}$ \\ ${ }^{a}$ IRII, Institut de Robòtica e Informàtica Industrial, Universitat Politècnica de Catalunya-Consejo \\ Superior de Investigaciones Científicas Parc Tecnològic de Barcelona - Edifici U, C/Llorens I Artigues, \\ 4-6, 2 , 08028, Barcelona, Spain; ${ }^{b}$ Electrical and Computer Engineering Department, The University \\ of Arizona, Tucson, AZ, 85721, USA
}

Two previous papers [Mirats et al. (2002a) "On the selection of variables for Qualitative Modelling of Dynamical Systems", International Journal of General Systems 31(5) pp. 435-467; Mirats et al. (2002b) "Variable selection procedures and efficient suboptimal mask search algorithms in Fuzzy Inductive Reasoning", International Journal of General Systems 31(5), pp. 469-498] were devoted to the selection of a set of variables that can best be used to model (reconstruct) a given output variable, whereby only static relations were analysed. Yet even after reducing the set of variables in this fashion, the number of remaining variables may still be formidable for large-scale systems. The present paper aims at tackling this problem by discovering substructures within the whole set of the system variables. Hence whereas previous research dealt with the problem of model reduction by means of reducing the set of variables to be considered for modelling, the present paper focuses on model structuring as a means to subdivide the overall modelling task into subtasks that are hopefully easier to handle. The second and third sections analyse this problem from a system-theoretic perspective, presenting the reconstruction analysis (RA) methodology, an informational approach to the problem of decomposing a large-scale system into subsystems. The fourth section uses the fuzzy inductive reasoning (FIR) methodology to find a possible structure of a system. The study performed in this paper only considers static relations.

Keywords: Model structuring, variable selection, behavioural modelling, model reduction, inductive modelling, fuzzy inductive reasoning

\section{INTRODUCTION}

Given a set of observations of the input/output behaviour of a physical system, it is desirable to obtain a behavioural model. Such models are needed for fault monitoring as well as intelligent control. A well-suited technique for qualitative behavioural modelling and simulation of physical systems is fuzzy inductive reasoning (FIR) (Cellier, 1991), a methodology based on general systems theory. Yet the FIR modelling methodology is of

\footnotetext{
*Corresponding author. Tel.: +34-934015791. Fax: +34-934015750. E-mail: jmirats@iri.upc.es; rhuber@iri.upc.es

†E-mail: cellier@ece.arizona.edu
} 
exponential computational complexity, and therefore it may be useful to consider other approaches as booster techniques for FIR.

Two previous papers (Mirats et al., 2000a,b) describing the last results on the FIR methodology looked at different variable selection techniques with the aim of identifying a subset of variables that are best suited for reconstructing (modelling) a given output variable. The different developed algorithms were applied to two different real systems, a steam generator and a garbage incinerator process. These methods can be classified as model reduction techniques and can be used as a precursor to the FIR modelling engine. Their aim is to reduce the FIR search space to make the FIR methodology better suited for dealing with large-scale systems. Yet even if the number of variables can be reduced dramatically by variable selection techniques, the set of remaining variables to be considered by the FIR modelling engine may still be far too large to allow the engine to converge within reasonable computation time.

Therefore, a second precursor to FIR modelling should be performed: using those variables that are retained in the previously performed variable selection step for modelling the system, subsets of variables need to be found that are maximally related among each other. These subsets can then be used to determine sub-models of the overall model. These kinds of techniques can be classified as model structuring techniques. The corresponding algorithms are often referred to in the literature as structure identification algorithms (Klir, 1985).

A question arises here: why is it desirable to represent a complex system by means of a collection of subsystems?

Several arguments may be stated. First, it may be either impractical or impossible to measure all the variables relevant to the modelling task at equal frequencies. Some variables may change over time much more slowly than others, and consequently should be sampled less frequently. If the data are collected in groups of related variables forming part of the same subsystem,, it is only necessary to measure each of those groups consistently and coherently. Second, even if all of the data were sampled at equal frequencies, storing all of them in a single table is impractical, because all combinations of states would then need to be recorded, even for variables that are almost uncorrelated with one another. The superset of all legal states of the overall system is much larger than the concatenation of sets of all legal states of all subsystems. Another important reason is related to the easiness of the process design when a subsystem decomposition of the complex system is available. This is a very common feature used in engineering design. The subdivision of the overall system into parts enables the designer to find solutions for each of these parts separately then connect the individual designs to obtain a (usually sub-optimal) design for the overall system. Another important issue to take into account is the number of sensors the system needs to be observed. Sensors may be expensive, and difficult to integrate within the system to observe, and it may be impossible to provide the system with sensors for all the variables. If a structure for such a system is derived, the number of sensors can be reduced only providing sensing devices for input variables, whereas the internal variables can be estimated using FIR models.

In the context of FIR, the primary motivation for subdividing a system into parts is similar to the last argument mentioned above. Let us assume that data are available to capture the dynamics of the entire system to be modelled. A subdivision of the system into parts (a subdivision of the set of variables into subsets) simplifies the FIR modelling task, and often makes an otherwise intractable problem tractable. Once the FIR models of the subsystems have been found, the behaviour of the overall system can be reconstructed from the individual behaviours of its parts.

\footnotetext{
${ }^{\ddagger}$ The reader may notice that, in general, the subsystems obtained by means of a decomposition method do not necessarily coincide with the physical subsystems of the complex system being investigated.
} 
This idea has its roots in the fact that, given a $k$-variables system, the cost of computing a unique $k$-variable model is much higher than computing a set of $p$ models of $j_{p}<k$ variables. Different authors have tackled this problem in the past from an information systems point of view. In Ashby (1965), it is stated that complex systems are unmanageable and should be decomposed into subsystems. Also in this work, some of the formulae measuring the information exchange between variables are presented. In Conant $(1972,1976)$, the measure $T$ of energy transference between variables is used to determine a probable subsystem decomposition. In Broekstra (1976, 1977), the information theory introduced previously by Ashby is used to decompose systems using modified procedures coined "constraint analysis" by the author. In Madden and Ashby (1972), relations between variables are offered that define the representation of an $N$-variable system in terms of a set of $P$-variables subsystems, where $P<N$. In fact, it is that study which lays the foundations of what later would be introduced by Cavallo and Klir as the Reconstructability Analysis (Cavallo and Klir, 1979a,b; 1981a,b). Gaines (1977) offers a different yet parallel point of view to that of Klir's general system problem solving (GSPS) approach. The problem of structure identification, i.e. identification of subsystems, is tackled in Broekstra (1978). In Kengerlinsky (1978), an approach for decomposing a system into subsystems based on the Shannon entropy measure is provided. In Krippendorff $(1981,1986)$, yet another technique for determining the structure of a system is given. He also provides a comparison of his approach to those of Klir, Conant, and Broekstra.

In this paper, two different methods for obtaining subsets (subsystems) of variables are given. A third method based on a combination of linear and non-linear statistical techniques was already given in Mirats and Verde (2000). The FIR methodology is not resumed again, in order to keep the paper in a readable size. However, a full description of the FIR methodology can be found in Cellier (1991) and Mirats (2002).

In the second section, the reconstruction analysis (RA) methodology, a GSPS ${ }^{\text {I }}$ level-4 tool, is presented. The basic idea of RA is that of identifying, among all possible decompositions of a system, the one from which the behaviour of the overall system can best be reconstructed. The best decomposition is the one that leads to a reconstructed system, the behaviour of which is most similar to the original system behaviour. One of the requirements of this methodology is that all system variables are present in the set of obtained subsystems, classifying RA as a model structuring technique. The third section hands in an RA-based algorithm that will help us to find a subsystem decomposition of a whole system.

In the fourth section, an FIR-based approach to model structuring is presented. Starting from the desired output variable, sequences of sub-models are constructed, until all variables are accounted for as either input variables or internal variables. The fifth section is devoted to assess the results by means of predicting the output of the studied system with data that were not used to derive the different structures.

Both methodologies presented in this chapter only deal with models of zero time delay, i.e. they only consider static relations among variables. The inclusion of time in the analysis, and the study of dynamic relations among the variables are left for a future research effort. The functioning of the different algorithms presented is illustrated by means of applying them to a real system: a garbage incinerator system described in the Appendix. By using the same example across multiple model structuring methodologies, the functioning of the set of algorithms becomes more transparent, and the reader is able to acquire a deeper understanding of the methodological underpinnings of the proposed algorithms.

\footnotetext{
"A complete description of the epistemological levels in the mark of Klir's GSPS (general systems problem solving) framework is provided in Klir (1985).
} 


\section{RECONSTRUCTION ANALYSIS}

RA was developed by Cavallo and Klir in the early eighties. It tackles two complementary problems associated with the relationship between a global system and the various sets of its subsystems. The first problem is referred to in the literature as the reconstruction problem. Given a global system, the aim is to determine which structure systems, each one based on a set of subsystems of the overall system, are adequate for reconstructing the global system with an acceptable level of approximation.

The other problem is what has been called in the literature the identification problem. That is, given a set of subsystems by means of their behaviours, known to form part of a global system, to find the possible global systems that embrace those subsystems, so inferences about the unknown global system can be made.

RA originated with the reconstructability analysis proposed in Cavallo and Klir (1979a,b; 1981a,b) and Klir (1981). The methodology was refined in Klir (1991), Cellier (1991), de Albornoz (1996) and Mirats (2002). RA is closely related to the FIR methodology, ${ }^{\S}$ because it deals with the system behaviour information expressed in qualitative terms that are either crisp or fuzzy. It allows identifying temporal causal structures of a system, i.e. it can be used to determine a subsystem decomposition of a system. Yet in practice it is not useful for systems with more than say a dozen variables due to its exponential computational complexity.

\subsection{The Concepts of Reconstructability}

Usually a model of a system is understood as a set of rules mapping a set of variables onto each other (Klir, 1985). If an input-output model of a system is desired, this set of rules maps all the related input (and possibly internal) variables to the considered output so that the behaviour of the system can be obtained from past data and this set of mapping rules. This is basically what the FIR methodology does.

This kind of reasoning may be impractical in the presence of large numbers of variables. In this case, it would be better to obtain a description of the internal structure of the system in the form of rules that are used inside the model to map variables onto each other. To find the structure of the system, in the most abstract case, means to search for a subsystem decomposition of this system, then obtain a model for each one of these subsystems separately, and finally integrate the information of those subsystems in a way that describes the global system. This is what the RA methodology strives to accomplish.

Reconstructability analysis is conceived as a package of methodological tools that deals with the problem of defining the relationship between a global system and its various subsystems. As briefly stated before, two main problems are tackled within this set of tools: the identification and the reconstruction problem. The tools needed to solve the identification problem are:

- Synthesis of the reconstruction family for a given set of subsystems. A reconstruction family is defined as the set of global systems that may be represented by the given set of subsystems in the sense that the behaviour of these subsystems may be obtained as a projection of the overall behaviour.

- Determination of the reconstruction uncertainty, or identifiability quotient. This metric, based on the size of the reconstruction family, is used to determine the uncertainty associated with the reconstruction of the global system from the given set of subsystems. Such a metric can be derived for either probabilistic or possibilistic systems.

\footnotetext{
${ }^{\S}$ Reconstruction analysis is fed with the same qualitative data used in the FIR methodology.
} 


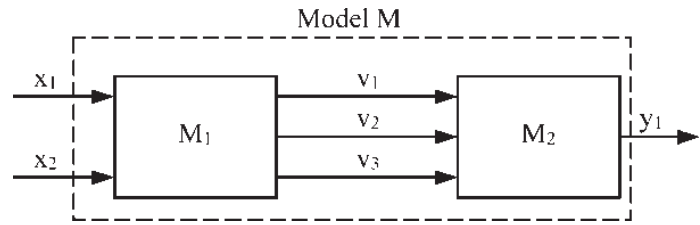

FIGURE 1 A model with two possible subsystems.

- Identification of the unbiased reconstruction. A unique global system is selected from the previously generated reconstruction family. The goal is to select a global system that contains, using Klir's words, "all, but no more information than is contained in the structure system." Unbiased here means that, from the global behaviour, the known set of subsystem behaviours should be obtained when projecting the global behaviour onto the set of variables forming each of the subsystems.

In the case of the reconstruction problem, the tools needed are:

- Generation of reconstruction hypotheses for a given global system. By reconstruction hypothesis is meant a possible set of subsystems from which the behaviour of the global system can be reconstructed.

- A computation of the desirable projections of the given behaviour system.

- Computation of the distances between the given behaviour and those reconstructed from each of the reconstruction hypotheses. This distance computation will allow establishing a rank order among the different considered reconstruction hypotheses.

\subsection{Structure Systems in Reconstruction Analysis}

Consider, for example, a model, $M$, of a six variables system that is formed from two submodels, $M_{1}$ and $M_{2}$. Figure 1 shows the topological structure of this system.

In the given example, the model is formed by six variables: two input variables, $x_{1}$ and $x_{2}$, being inputs of both the model $M$ and the first submodel $M_{1}$; three internal variables, named $v_{1}, v_{2}$, and $v_{3}$, being the outputs of the first submodel $M_{1}$ as well as the inputs to the second submodel $M_{2}$; and one output variable, $y_{1}$, that is the output of the second submodel $M_{2}$ as well as of the model $M$.

The structure can be abstracted in RA by means of a so-called composite structure. A composite structure is a row vector, in which each element enumerates a variable of a subsystem, and different substructures are separated by 0 elements.

$$
\mathrm{CST}^{1}=\left(\begin{array}{llllllllll}
1 & 2 & 3 & 4 & 5 & 0 & 3 & 4 & 5 & 6
\end{array}\right) .
$$

$\mathrm{CST}^{1}$ is the composite structure of the example in Fig. 1, where the variables have been labelled from 1 to 6 , beginning with $x_{1}$ and finishing with $y_{1}\left(x_{1}=1, x_{2}=2, v_{1}=3, v_{2}=4\right.$, $v_{3}=4, y_{1}=6$ ).

The reader may notice that, at this level of the methodology, no distinction is made any longer between inputs and outputs, thus no causality is expressed by the composite structure."

\footnotetext{
${ }^{\|}$The causality of a mathematical model of a physical system is an artifact of the way in which models are commonly used by simulation code, rather than being a property of the physical system itself. Hence it is meaningful to offer a mathematical description of the system structure that reflects physical reality by not forcing the user to provide causality information.
} 
Variables are simply labelled with an integer number and enumerated one by one as they are encountered in a submodel. The abstraction of a topological structure into a composite structure is unique and straightforward. The opposite is not true. A single composite structure may be representative of zero, one, or multiple topological structures.

Another way of expressing these kinds of structures, also used in RA, is by means of the so-called binary structure. It consists of an ordered enumeration of all the possible binary relations or connections among variables inside all subsystems. $\mathrm{BST}^{1}$ expresses the corresponding binary structure in the example given in Fig. 1. Notice that, of all possible connections among the six system variables, only two relations are missing, namely the connections $(1,6)$ and $(2,6)$, indicating that there do not exist direct connections between variables 1, 2 (the input variables of the system) on the one hand, and variable 6 (the output variable of the system) on the other.

There exist two special cases, the totally unconnected model and the totally connected model. The former is a model that contains a set of unrelated variables. Since there are no connections among variables, its binary structure is empty. The latter is a model without any internal structure. Every variable is related to every other variable. Consequently, its binary structure is complete.

$$
\mathrm{BST}^{1}=\left(\begin{array}{cc}
1 & 2 \\
1 & 3 \\
1 & 4 \\
1 & 5 \\
2 & 3 \\
2 & 4 \\
2 & 5 \\
3 & 4 \\
3 & 5 \\
3 & 6 \\
4 & 5 \\
4 & 6 \\
5 & 6
\end{array}\right) .
$$

\subsection{Tools available in Reconstruction Analysis}

Given an observed behaviour, the first step on the way to solving the structure identification problem is the generation of reconstruction hypotheses. In RA, the behaviour is defined in exactly the same fashion as it was done for the FIR methodology." A reconstruction hypothesis is simply a proposed composite structure over the set of variables contained in the behaviour. Once a reconstruction hypothesis has been formulated, the behaviour of the global system is projected onto each of the subsystems obtaining the subsystem behaviours. The behaviours of the individual subsystems are then recombined, leading to a reconstructed overall behaviour. The quality of the reconstruction is evaluated by

${ }^{\#}$ The behaviour is expressed by means of a matrix that contains the ordered set of all observed states of the system together with a confidence vector expressing the likelihood of each state to occur. 


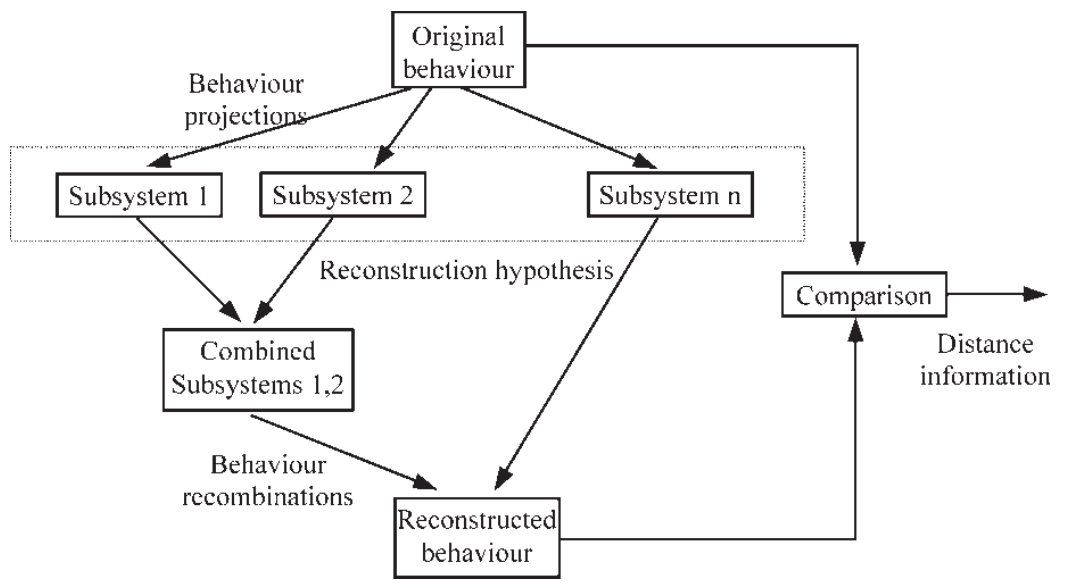

FIGURE 2 Reconstruction evaluation process.

measuring the distance between the original system behaviour and the reconstructed one. Figure 2 shows this process. A full description of how this process is performed can be found in Mirats (2002).

\subsubsection{Algorithms to Generate Reconstruction Hypotheses}

In order to find the optimal subsystem decomposition using FRA, all possible sets of subsystems of a given global system should be computed. This is a formidable task, feasible only for small-scale systems comprised of few variables. The number of possible subsystem decompositions grows exponentially with the number of variables. Three sub-optimal search algorithms have been reported in the open literature (Uyttenhove, 1978; Klir and Uyttenhove, 1979; Cavallo and Klir, 1979b; 1981b) that usually generate satisfactory system decompositions.

The first of these algorithms has been called structure refinement. It starts out with the totally connected binary structure, i.e. all possible binary relations between the system variables are considered. For the given example, the totally connected composite structure is defined as:

$$
\mathrm{CST}^{2}=\left(\begin{array}{llllll}
1 & 2 & 3 & 4 & 5 & 6
\end{array}\right) .
$$

The totally connected structure has no reconstruction error, since no reconstruction needs to be done. Then, at each step, one binary connection is severed at a time, and the reconstruction error for the resulting subsystem decomposition is computed. When for the present level of decomposition all binary connections have been severed one at a time, the one that results in the structure with the smallest reconstruction error is permanently removed. At the first step of the algorithm, the number of reconstructions that is to be computed in a $k$-variable system is given by:

$$
\frac{k(k-1)}{2} .
$$

Then a second binary relation is severed. The algorithm follows on severing one binary relation at a time, until the one with the smallest reconstruction error has been found. The corresponding binary relation is then deleted permanently. The algorithm continues in the same fashion, until the reconstruction error of all of the candidate structures at the next level becomes larger than the limit imposed by the investigator. A quality measure based on 
the reconstruction error is introduced.

$$
Q_{i}=1-\frac{\text { rec_err }}{i}
$$

where rec_err ${ }_{i}$ is the reconstruction error associated with the $i$ th reconstruction hypothesis, and rec_err tot_unc is the reconstruction error of the totally unconnected structure, which exhibits the largest reconstruction error possible. The quality measure is a metric normalized to the range [0,1], where higher quality values denote better reconstruction hypotheses. For the example given in Fig. 1, the totally unconnected structure is defined as:

$$
\mathrm{CST}^{3}=\left(\begin{array}{lllllllllll}
1 & 0 & 2 & 0 & 3 & 0 & 4 & 0 & 5 & 0 & 6
\end{array}\right) .
$$

When the structure refinement algorithm is applied to the example at hand (for the system given in Fig. 1, an artificial behaviour matrix and a confidence vector are used to obtain the given results in this section. Those are not reported here; refer to Mirats (2002)) with a smallest tolerated quality of $Q_{\min }=0.1$, the following result is obtained:

$$
\text { RESULTANT STRUCTURE: (1,6) (2) (3) (4) (5). }
$$

The second algorithm found in the open literature is the so-called structure aggregation algorithm. The strategy followed here is just the opposite of the one followed in the previously described algorithm. In this case, the algorithm starts out with the totally unconnected model, and at each time step, one binary connection is added. From all possible structure candidates, the one that most reduces the reconstruction error is considered the best, i.e. the binary relation that, when added, most reduces the reconstruction error is added permanently. The algorithm ends when the reconstruction error drops below the largest tolerable error specified by the modeller. For the given example, this algorithm, with $Q_{\min }=0.1$, leads to the following results:

$$
\text { RESULTANT STRUCTURE: }(1,3) \text { (1,6) (2) (4) }(5) \text {. }
$$

The structures resulting from the application of the two algorithms are not identical, but similar. In the given example, the structure aggregation algorithm converged much faster, because the selected minimum quality was very low. If a higher value of $Q_{\min }$ had been selected, the structure refinement algorithm would have converged faster, whereas the structure aggregation algorithm would have required more time to reach the desired goal.

Finally, the third of the known sub-optimal search strategies is the so-called single-step refinement algorithm. This algorithm, just like the refinement algorithm, starts out with the totally connected structure. The first step of the single-step refinement algorithm is identical to that of the refinement algorithm. However, at the end of the first step, all relations are permanently severed that exhibit a reconstruction error that is smaller than the largest tolerated one, or in the current implementation, that exhibit a quality that is better than the lowest permissible one. Only a single step of the algorithm is performed. Whereas the refinement algorithm and the aggregation algorithm are still of exponential complexity (though much faster than exhaustive search), the single-step refinement algorithm is of polynomial complexity.

When this algorithm is applied to the given example, allowing a largest reconstruction error of $e_{\max }=0.005$ (please refer to Mirats et al. (2002) for a definition of the reconstruction error) the following results are obtained: 


\section{APPLICATION OF FRA IN CONJUNCTION WITH A VARIABLE SELECTION TECHNIQUE}

In this section, FRA is applied to the garbage incinerator system briefly described in the Appendix, but only involving the 15 variables remaining from a correlation analysis performed so as to eliminate redundant variables (Mirats and Verde, 2000). With this pre-filtering of the system variables, it is possible to reduce the number of structures that FRA needs to investigate to determine the internal structure of the system. In this way, the computing time of the FRA engine can be significantly reduced when searching for a plausible structure within the set of system variables. The discarded variables were $x_{1}, x_{3}, x_{15}, x_{16}$, and $x_{17}$, so 14 input variables and of course the output one remain for the analysis. Due to the size of the system, only the single-step refinement algorithm has been used.

Up to 43200 data vectors are available from the incinerator system, 42700 were used to compute the models and the last 500 were left to assess the results. The single-step refinement algorithm required roughly $11 \mathrm{~h}$ of computing time on a Sun Ultra-Sparc II. The results from this experiment are listed in Table I.

BINARY RELATIONS WITH AN ERROR LARGER THAN 0.1000 ARE CONSIDERED

RESULTANT STRUCTURE: (2) (4) (5) (6) (7) (8) (9) (10) (11) (12) (13) (14) (18) (19) (20)

BINARY RELATIONS WITH AN ERROR LARGER THAN 0.0100 ARE CONSIDERED

RESULTANT STRUCTURE: $(5,8,20)(5,11,14)(7,8,20)(7,14,18)(8,10,20)(10,14,18)(2,8,9,14)$

$(4,6,7,8,19)(4,6,7,12,19)(4,9,11,12,13)(2,9,11,12,13,14)(4,6,7,9,12,13)$

BINARY RELATIONS WITH AN ERROR LARGER THAN 0.0150 ARE CONSIDERED

RESULTANT STRUCTURE: $(4,10)(10,13)(10,18)(14,18)(2,11,13)(2,12,13)(4,6,12)(4,6,19)(5,11,14)$ $(8,10,20)(9,12,13)(6,7,8,19)(6,8,9,12)(7,8,12,14)(7,12,13,14)$.

Three different largest reconstruction error values were applied: $e_{\max }=0.1, e_{\max }=0.01$, and $e_{\max }=0.015$. Analysing the results of this experiment, when the largest reconstruction error of a single binary relation between variables to be omitted is set to $e_{\max }=0.1$, the algorithm does not find any binary relation to consider: all of them are omitted, and a totally unconnected structure is obtained.

In contrast with $e_{\max }=0.01,55$ out of the 105 possible binary relations are considered. The resulting subsystems, labelled $\mathrm{S} 1_{\mathrm{FRA} 0.01}$ to $\mathrm{S} 12_{\mathrm{FRA} 0.01}$, are listed in Table II. Unfortunately, the result is difficult to interpret. Three different structures contain the system output, $X_{20}$. Which of those should be used to compute the output? Do the other two occurrences introduce feedback loops into the resulting topological structure? How are algebraic loops among variables avoided?

To avoid this problem, the largest reconstruction error was increased to $e_{\max }=0.015$. In this case, 36 of the 105 binary relations are retained, and only one subsystem containing the output variable is found. The resulting substructures are listed in Table III.

The output is computed using variables $X_{8}$ and $X_{10}$. How are those variables evaluated? Unfortunately, both $X_{8}$ and $X_{10}$ appear in three of the remaining substructures. Which of those should be used to compute them? What about feedback structures? Quite evidently, the previously mentioned causality problem has not been solved, only delayed. 
TABLE I Single step refinement algorithm results

\begin{tabular}{|c|c|c|c|}
\hline Error & Binary relation omitted & Error & Binary relation omitted \\
\hline 0.000121 & 2,4 & 0.000221 & 7,11 \\
\hline 0.000031 & 2,5 & 0.022214 & 7,12 \\
\hline 0.000024 & 2,6 & 0.016339 & 7,13 \\
\hline 0.000011 & 2,7 & 0.020031 & 7,14 \\
\hline 0.011050 & 2,8 & 0.013191 & 7,18 \\
\hline 0.014203 & 2,9 & 0.021365 & 7,19 \\
\hline 0.000042 & 2,10 & 0.014242 & 7,20 \\
\hline 0.021082 & 2,11 & 0.023871 & 8,9 \\
\hline 0.016028 & 2,12 & 0.018399 & 8,10 \\
\hline 0.027111 & 2,13 & 0.000102 & 8,11 \\
\hline 0.010561 & 2,14 & 0.000115 & 8,12 \\
\hline 0.008524 & 2,18 & 0.006032 & 8,13 \\
\hline 0.000286 & 2,19 & 0.023143 & 8,14 \\
\hline 0.009398 & 2,20 & 0.000282 & 8,18 \\
\hline 0.000033 & 4,5 & 0.018324 & 8,19 \\
\hline 0.028183 & 4,6 & 0.018102 & 8,20 \\
\hline 0.012103 & 4,7 & 0.000067 & 9,10 \\
\hline 0.013151 & 4,8 & 0.014634 & 9,11 \\
\hline 0.012272 & 4,9 & 0.015811 & 9,12 \\
\hline 0.016134 & 4,10 & 0.018401 & 9,13 \\
\hline 0.014261 & 4,11 & 0.014753 & 9,14 \\
\hline 0.025047 & 4,12 & 0.000297 & 9,18 \\
\hline 0.013029 & 4,13 & 0.000214 & 9,19 \\
\hline 0.008572 & 4,14 & 0.000216 & 9,20 \\
\hline 0.006091 & 4,18 & 0.008464 & 10,11 \\
\hline 0.016835 & 4,19 & 0.015678 & 10,12 \\
\hline 0.005103 & 4,20 & 0.018031 & 10,13 \\
\hline 0.000067 & 5,6 & 0.013597 & 10,14 \\
\hline 0.000026 & 5,7 & 0.023159 & 10,18 \\
\hline 0.013272 & 5,8 & 0.000157 & 10,19 \\
\hline 0.000661 & 5,9 & 0.021783 & 10,20 \\
\hline 0.000017 & 5,10 & 0.014957 & 11,12 \\
\hline 0.021411 & 5,11 & 0.019011 & 11,13 \\
\hline 0.000019 & 5,12 & 0.021164 & 11,14 \\
\hline 0.000011 & 5,13 & 0.008869 & 11,18 \\
\hline 0.015141 & 5,14 & 0.008357 & 11,19 \\
\hline 0.004083 & 5,18 & 0.008176 & 11,20 \\
\hline 0.000075 & 5,19 & 0.024819 & 12,13 \\
\hline 0.011106 & 5,20 & 0.031104 & 12,14 \\
\hline 0.024196 & 6,7 & 0.000116 & 12,18 \\
\hline 0.022142 & 6,8 & 0.011067 & 12,19 \\
\hline 0.015137 & 6,9 & 0.000258 & 12,20 \\
\hline 0.000028 & 6,10 & 0.023164 & 13,14 \\
\hline 0.000001 & 6,11 & 0.000103 & 13,18 \\
\hline 0.023185 & 6,12 & 0.000037 & 13,19 \\
\hline 0.014601 & 6,13 & 0.000135 & 13,20 \\
\hline 0.000084 & 6,14 & 0.021305 & 14,18 \\
\hline 0.000079 & 6,18 & 0.000109 & 14,19 \\
\hline 0.031102 & 6,19 & 0.000099 & 14,20 \\
\hline 0.000174 & 6,20 & 0.002862 & 18,19 \\
\hline 0.021566 & 7,8 & 0.004012 & 18,20 \\
\hline 0.012323 & 7,9 & 0.002243 & 19,20 \\
\hline 0.000367 & 7,10 & & \\
\hline
\end{tabular}

\subsection{Obtaining a Topological Structure of a System Using a New FRA-based Algorithm}

Talking about the internal structure of a system using FRA-based algorithms three different structure types have been introduced:

- The topological structure, represented by a block diagram showing subsystems with inputs and outputs as well as interconnections between them. 
TABLE II Incinerator subsystem decomposition obtained with FRA using $e_{\max }=0.01$

\begin{tabular}{lcccccccccc}
\hline $\mathrm{S} 1_{\text {FRA0.01 }}$ & $\mathrm{X}_{5}$ & $\mathrm{X}_{8}$ & $\mathrm{X}_{20}$ & $\mathrm{~S} 7_{\text {FRA0.01 }}$ & $\mathrm{X}_{2}$ & $\mathrm{X}_{8}$ & $\mathrm{X}_{9}$ & $\mathrm{X}_{14}$ & & \\
$\mathrm{~S} 2_{\text {FRA0.01 }}$ & $X_{5}$ & $X_{11}$ & $X_{14}$ & $\mathrm{~S} 8_{\text {FRA0.01 }}$ & $X_{4}$ & $X_{6}$ & $X_{7}$ & $X_{8}$ & $X_{19}$ & \\
$\mathrm{~S} 3_{\text {FRA0.01 }}$ & $X_{7}$ & $X_{8}$ & $X_{20}$ & $\mathrm{~S} 9_{\text {FRA0.01 }}$ & $X_{4}$ & $X_{6}$ & $X_{7}$ & $X_{12}$ & $X_{19}$ & \\
$\mathrm{~S} 4_{\text {FRA0.01 }}$ & $X_{7}$ & $X_{14}$ & $X_{18}$ & $\mathrm{~S} 10_{\text {FRA0.01 }}$ & $X_{4}$ & $X_{9}$ & $X_{11}$ & $X_{12}$ & $X_{13}$ & \\
$\mathrm{~S} 5_{\text {FRA0.01 }}$ & $X_{8}$ & $X_{10}$ & $X_{20}$ & $\mathrm{~S} 11_{\text {FRA0.01 }}$ & $X_{2}$ & $X_{9}$ & $X_{11}$ & $X_{12}$ & $X_{13}$ & $X_{14}$ \\
S6 $6_{\text {FRA0.01 }}$ & $X_{10}$ & $X_{14}$ & $X_{18}$ & S12 $2_{\text {FRA0.01 }}$ & $X_{4}$ & $X_{6}$ & $X_{7}$ & $X_{9}$ & $X_{12}$ & $X_{13}$ \\
\hline
\end{tabular}

- The composite structure, obtained from the topological structure by enumerating the variables of each subsystem, ignoring whether they are input or output, and ignoring the topology of connections between the subsystems.

- The binary structure, obtained from the composite structure by expanding the variables of each subsystem into a set of binary relations and then merging all these sets into one in order to eliminate the redundant binary relations.

The conversion from the topological structure via the composite structure to the binary structure is unique. Unfortunately the conversion from the binary to the composite structure is not unique, and we need additional rules to decide which of the possible composite structures is to be selected. The conversion from the composite structure to the topological structure is not unique either. There may exist 0,1 , or multiple topological structures representing the same composite structure.

Whereas the binary structure is most useful for searching through a structure space and for comparing different structures with each other, what we ultimately need for a practical realisation is the topological structure. The single-step refinement algorithm of FRA will give us the relative strengths of all binary relations. It can be used to derive different binary structures, which then in turn can be converted to a composite structure. Unfortunately, there is no known way to obtain a topological structure from that.

Since we need a topological structure, a different FRA-based algorithm is now proposed that can be used to derive the topological structure directly. It is based on the observation that the complete connectedness of a substructure only remains important as long as no causality is attached to it. Once it has been decided which variable needs to be computed from the substructure, it is only important that the binary relations between the inputs and that output are strong. The binary relations among the different inputs are no longer of any major concern. In fact, it might be preferable that they are weak so that the model does not operate on unnecessary redundant information. The algorithm works as follows.

- We look at the relative strengths of all binary relations with the output variable. Those relations with strength larger than $x$ are identified, where $x$ may assume a value such as 0.01. For the example at hand, i.e. the incinerator system, we find the following

TABLE III Incinerator subsystem decomposition obtained with FRA using $e_{\max }=0.015$

\begin{tabular}{|c|c|c|c|c|c|c|c|c|}
\hline $\mathrm{S} 1_{\text {FRA0.015 }}$ & $\mathrm{X}_{4}$ & $\mathrm{X}_{10}$ & & $\mathrm{~S} 9_{\text {FRA0.015 }}$ & $\mathrm{X}_{5}$ & $\mathrm{X}_{11}$ & $\mathrm{X}_{14}$ & \\
\hline $\mathrm{S} 2_{\mathrm{FRA} 0.015}$ & $X_{10}$ & $X_{13}$ & & $\mathrm{~S} 10_{\mathrm{FRA} 0.015}$ & $X_{8}$ & $X_{10}$ & $X_{20}$ & \\
\hline $\mathrm{S} 3_{\mathrm{FRA} 0.015}$ & $X_{10}$ & $X_{18}$ & & $\mathrm{~S} 11_{\text {FRA0.015 }}$ & $X_{9}$ & $X_{12}$ & $X_{13}$ & \\
\hline $\mathrm{S} 4_{\mathrm{FRA} 0.015}$ & $X_{14}$ & $X_{18}$ & & $\mathrm{~S} 12_{\text {FRA0.015 }}$ & $X_{6}$ & $X_{7}$ & $X_{8}$ & $X_{19}$ \\
\hline $\mathrm{S} 5_{\text {FRA0.015 }}$ & $X_{2}$ & $X_{11}$ & $X_{13}$ & $\mathrm{~S} 13_{\text {FRA0.015 }}$ & $X_{6}$ & $X_{8}$ & $X_{9}$ & $X_{12}$ \\
\hline S6 $6_{\text {FRA0.015 }}$ & $X_{2}$ & $X_{12}$ & $X_{13}$ & $\mathrm{~S} 14_{\text {FRA0.015 }}$ & $X_{7}$ & $X_{8}$ & $X_{12}$ & $X_{14}$ \\
\hline $\mathrm{S} 7_{\mathrm{FRA} 0.015}$ & $X_{4}$ & $X_{6}$ & $X_{12}$ & $\mathrm{~S} 15_{\mathrm{FRA} 0.015}$ & $X_{7}$ & $X_{12}$ & $X_{13}$ & $X_{14}$ \\
\hline $\mathrm{S} 8_{\text {FRA0.015 }}$ & $\mathrm{X}_{4}$ & $\mathrm{X}_{6}$ & $\mathrm{X}_{19}$ & & & & & \\
\hline
\end{tabular}


significantly large binary relations with the output (the binary relations are listed using the indices of the variables only):

\begin{tabular}{ll}
\hline 5,20 & err $=0.011106$ \\
7,20 & err $=0.014242$ \\
8,20 & err $=0.018102$ \\
10,20 & err $=0.021783$
\end{tabular}

- Since individual submodels that are too complex are not desired, the four most important inputs are selected, if there are at least four inputs with strengths greater than $x$, otherwise all inputs with strengths greater than $x$ are selected. For the case of the incinerator system, exactly four binary relations with the output variable are found that comply with these requirements. Consequently, the first substructure is found to be:

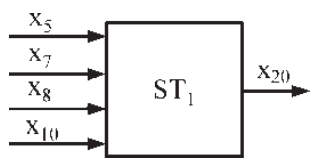

- The algorithm is repeated for every one of these inputs, excluding relations with those variables that had previously been used as outputs of subsystems. In the current situation, this only applies to $X_{20}$. The following substructures are found:

\begin{tabular}{ll}
\hline 5,8 & err $=0.013272$ \\
5,11 & err $=0.021411$ \\
5,14 & err $=0.015141$ \\
\hline
\end{tabular}

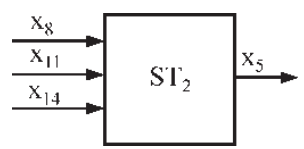

\begin{tabular}{ll}
\hline 4,7 & err $=0.012103$ \\
$\mathbf{6 , 7}$ & err $=\mathbf{0 . 0 2 4 1 9 6}$ \\
$\mathbf{7 , 8}$ & err $=\mathbf{0 . 0 2 1 5 6 6}$ \\
\hline
\end{tabular}

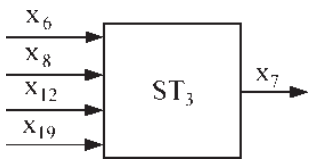

\begin{tabular}{ll}
\hline 7,9 & err $=0.012323$ \\
$\mathbf{7 , 1 2}$ & err $=\mathbf{0 . 0 2 2 2 1 4}$ \\
7,13 & err $=0.016339$ \\
7,14 & err $=0.020031$ \\
7,18 & err $=0.013191$ \\
$\mathbf{7 , 1 9}$ & err $=\mathbf{0 . 0 2 1 3 6 5}$ \\
\hline
\end{tabular}




\begin{tabular}{ll}
\hline 2,8 & err $=0.011050$ \\
4,8 & err $=0.013151$ \\
5,8 & err $=0.013272$ \\
$\mathbf{6 , 8}$ & err $=\mathbf{0 . 0 2 2 1 4 2}$ \\
$\mathbf{7 , 8}$ & err $=\mathbf{0 . 0 2 1 5 6 6}$ \\
$\mathbf{8 , 9}$ & err $=\mathbf{0 . 0 2 3 8 7 1}$ \\
8,10 & err $=0.018399$ \\
$\mathbf{8 , 1 4}$ & err $=\mathbf{0 . 0 2 3 1 4 3}$ \\
8,19 & err $=0.018324$ \\
\hline
\end{tabular}

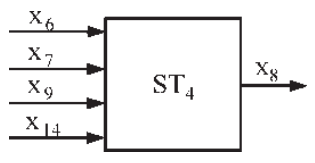

\begin{tabular}{ll}
\hline $\mathbf{4 , 1 0}$ & err $=\mathbf{0 . 0 1 6 1 3 4}$ \\
$\mathbf{8 , 1 0}$ & err $=\mathbf{0 . 0 1 8 3 9 9}$ \\
10,12 & err $=0.015678$ \\
$\mathbf{1 0}, \mathbf{1 3}$ & err $=\mathbf{0 . 0 1 8 0 3 1}$ \\
10,14 & err $=0.013597$ \\
$\mathbf{1 0}, \mathbf{1 8}$ & err $=\mathbf{0 . 0 2 3 1 5 9}$
\end{tabular}

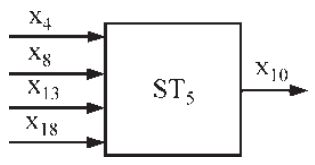

- Now it is necessary to eliminate algebraic loops. There is one algebraic loop in this example, because $X_{8}=f\left(X_{7}\right)$ and $X_{7}=f\left(X_{8}\right)$. The largest not selected relation among those of the two substructures is identified under the constraint to be different from the one that provokes the algebraic loop. For our example, we find the relation $(7,14)$. The algebraic loop relation is substituted by the new one, thus the $\mathrm{ST}_{3}$ substructure is replaced by:

\begin{tabular}{ll}
\hline 4,7 & err $=0.012103$ \\
7,8 & err $=0.021566$ \\
7,9 & err $=0.012323$ \\
7,13 & err $=0.016339$ \\
$\mathbf{7 , 1 4}$ & err $=\mathbf{0 . 0 2 0 0 3 1}$ \\
7,18 & err $=0.013191$ \\
\hline
\end{tabular}

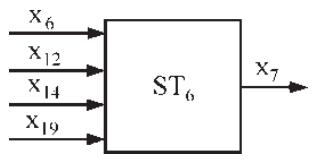

- Now, the variables that have not been used in any of the found substructures need to be studied. For the given example all variables except $X_{2}$ have been used. Thus, the strengths of all variables that have not yet been used as outputs can be checked with that variable:

\begin{tabular}{ll}
\hline 2,9 & err $=0.014203$ \\
2,11 & err $=0.021082$ \\
2,12 & err $=0.016028$ \\
$\mathbf{2 , 1 3}$ & err $=\mathbf{0 . 0 2 7 1 1 1}$ \\
2,14 & err $=0.010561$ \\
\hline
\end{tabular}

- The strongest relation is with variable $X_{13}$, thus we make a model of that variable:

\begin{tabular}{ll}
\hline $\mathbf{2 , 1 3}$ & err $=\mathbf{0 . 0 2 7 1 1 1}$ \\
4,13 & err $=0.013029$ \\
6,13 & err $=0.014601$ \\
9,13 & err $=0.018401$ \\
$\mathbf{1 1}, \mathbf{1 3}$ & err $=\mathbf{0 . 0 1 9 0 1 1}$ \\
$\mathbf{1 2}, \mathbf{1 3}$ & err $=\mathbf{0 . 0 2 4 8 1 9}$ \\
$\mathbf{1 3}, \mathbf{1 4}$ & err $=\mathbf{0 . 0 2 3 1 6 4}$
\end{tabular}

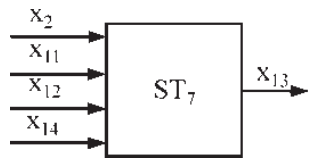




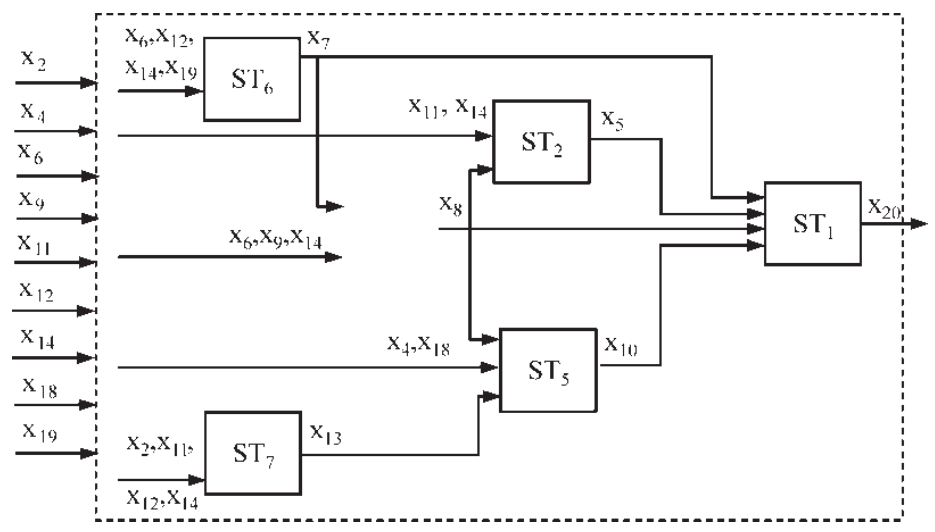

FIGURE 3 Topological structure obtained by means of an FRA-based algorithm.

By now a complete topological structure has been extracted from the single-refinement information of FRA. Before we had 15 variables, thus in order to model the output $\left(X_{20}\right)$ from the full set of inputs, we would have needed to use 14 sensors within the system. By now, we have six submodels. Each of them computes one variable, thus we only need nine sensors now. We could easily continue adding more substructures, until the number of sensors has been reduced to the desired level. Of course, these additional models will be poorer and poorer in quality, because there may be strong relations with previously used outputs that cannot be used again. Thus, there is a compromise to be made. Figure 3 shows the resulting structure of applying this algorithm to the incinerator system.

The proposed methodology offers a comprehensive approach to determining the substructure of a model, assuming that all variables need to be used (which makes sense, because these are the variables left over after the elimination step of Mirats and Verde (2000)). Once a structure has been found that accounts for all the variables, additional substructures can be added to reduce the number of true system inputs, i.e. the number of sensors that need to be used in the system. However, additional substructures should be added sparingly and after serious contemplation only, as these substructures will inevitably exhibit poorer tracking capabilities.

The single-step refinement algorithm found 55 important binary relations. The structure resulting from the proposed algorithm contains 46 binary relations, 37 of which are important, and nine are unimportant.

\section{USING FIR TO FIND THE STRUCTURE OF A SYSTEM}

Up to this point, FRA has been proposed as a technique for identifying the internal structure of a system. It was shown that its single-step refinement algorithm could indeed provide the information needed to determine a meaningful internal structure of a system. Unfortunately, the algorithm, at least in its current Matlab implementation, is far too slow to be used on a truly large-scale system.

An alternative algorithm based on FIR is proposed to derive the internal structure of a system. FIR is more efficient than FRA, and therefore it is hoped that similarly good results can be obtained considerably faster using this methodology. The algorithm follows similar paths of reasoning as the previously introduced FRA-based algorithm. As before, with the data of the garbage incinerator system, the algorithm is applied to the subset of variables remaining after the variable elimination step reported in Mirats and Verde (2000). 
In the sequel, the FIR-based structure identification algorithm is presented.

- With the considered variables, a flat (static) FIR model of the output variable is constructed. Due to the complexity issue, FIR will select only a subset of the possible inputs, probably not more than four or five of them. For the example at hand input variables $X_{2}, X_{4}, X_{5}, X_{6}, X_{7}, X_{8}, X_{9}, X_{10}, X_{11}, X_{12}, X_{13}, X_{14}, X_{18}, X_{19}$, and the output variable $X_{20}$ are being considered. When an optimal mask search is performed for this 15 variables system, FIR chooses the following model:

$$
X_{20}=f_{1}\left(X_{4}, X_{5}, X_{6}, X_{12}, X_{19}\right) \quad Q=0.2089 .
$$

Although we already know that $X_{20}$ depends on all of the remaining 14 inputs, FIR only selects a subset of those variables, namely the ones that are most useful in interpreting the observations made about the output. The reader may notice that the set of variables selected by FIR is dramatically different from the one chosen by FRA. The two models have only one variable, $X_{5}$, in common. The variable with the strongest binary relation to the output, $X_{10}$, was not selected at all by FIR.

- Now it is necessary to determine the relative importance of each of the inputs used by the model that was previously found. In the given example, there are five inputs. The relative importance of the five inputs could, of course, be determined using FRA, but the same can also be accomplished using FIR in the following manner. One at a time, each of the five inputs is severed, and the quality of the resulting FIR model without the severed input is computed. Thus, in the case at hand, the fuzzy quality of the five following models is computed:

$$
\begin{aligned}
& X_{20}=f_{2}\left(X_{4}, X_{5}, X_{6}, X_{19}\right) \rightarrow Q=0.1752 \\
& X_{20}=f_{3}\left(X_{4}, X_{5}, X_{12}, X_{19}\right) \rightarrow Q=0.1735 \\
& X_{20}=f_{4}\left(X_{5}, X_{6}, X_{12}, X_{19}\right) \rightarrow Q=0.1521 \\
& X_{20}=f_{5}\left(X_{4}, X_{6}, X_{12}, X_{19}\right) \rightarrow Q=0.1339 \\
& X_{20}=f_{6}\left(X_{4}, X_{5}, X_{6}, X_{12}\right) \rightarrow Q=0.1165 .
\end{aligned}
$$

Now, we have a measure of the importance of each of the inputs that model the output variable of the system. Since $f_{6}$ reduces the quality most, $X_{19}$ was the most important input. Similarly $X_{12}$ is the least important one.

- In this next step, an FIR model is generated for each of the inputs, starting with the least important one, excluding those variables as possible inputs that had previously been used as outputs. In the current situation, only $X_{20}$ needs to be excluded. The least important input for the incinerator system is $X_{12}$, and the FIR model found for this variable is:

$$
X_{12}=f_{7}\left(X_{4}, X_{6}, X_{7}, X_{9}, X_{13}\right) \quad \text { with } Q=0.4690
$$

Thus, we just found the next substructure.

- Now, we proceed with the next least important input of $f_{1}$, which happens to be $X_{6}$. Thus we make an FIR model, excluding all of those variables that were already outputs, namely $X_{12}$ and obviously $X_{20}$. In this way, we find the next substructure. The procedure continues in the same fashion until all physical inputs of the system, in this case 14, 


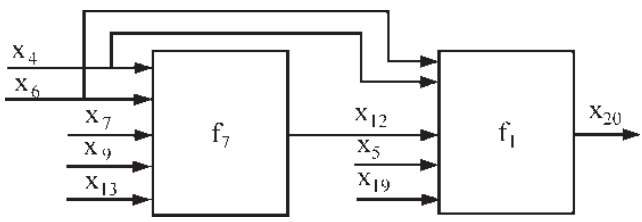

FIGURE 4 Second substructure found for the incinerator system using FIR.

appear among the inputs of the model. This gives us a complete structure. For the incinerator system, the following models are found for each of the inputs of the $f_{1}$ model:

$$
\begin{aligned}
& X_{6}=f_{8}\left(X_{4}, X_{7}, X_{8}, X_{13}\right) \quad \text { with } Q=0.4922 \\
& X_{4}=f_{9}\left(X_{2}, X_{9}, X_{13}, X_{14}, X_{19}\right) \quad \text { with } Q=0.2765 \\
& X_{5}=f_{10}\left(X_{8}, X_{10}, X_{11}, X_{14}, X_{18}\right) \text { with } Q=0.4696 \\
& X_{19}=f_{11}\left(X_{7}, X_{8}, X_{11}, X_{14}\right) \quad \text { with } Q=0.1375 .
\end{aligned}
$$

The given algorithm is based on the idea that it may not be economical to provide the system with sensors for all of the inputs (in this case, fourteen input variables). Instead, by providing an internal structure, sensors need only be provided for the true inputs, whereas the internal variables (such as $X_{12}$ in Fig. 4) can be estimated using FIR models. The least important inputs were modelled first, in order to reserve sensors for the more important ones. The resulting structure of applying this algorithm to the incinerator system is given in Fig. 5 .

The proposed methodology offers an alternative approach to determine the substructure of a model, assuming again that all variables need to be used. Just as in the previous case, once a structure has been found that accounts for all the variables, additional substructures can be added to reduce the number of true system inputs, i.e. the number of sensors that need to be used in the system. However, additional substructures should be added sparingly and after serious contemplation only, as these substructures will inevitably exhibit poorer tracking capabilities.

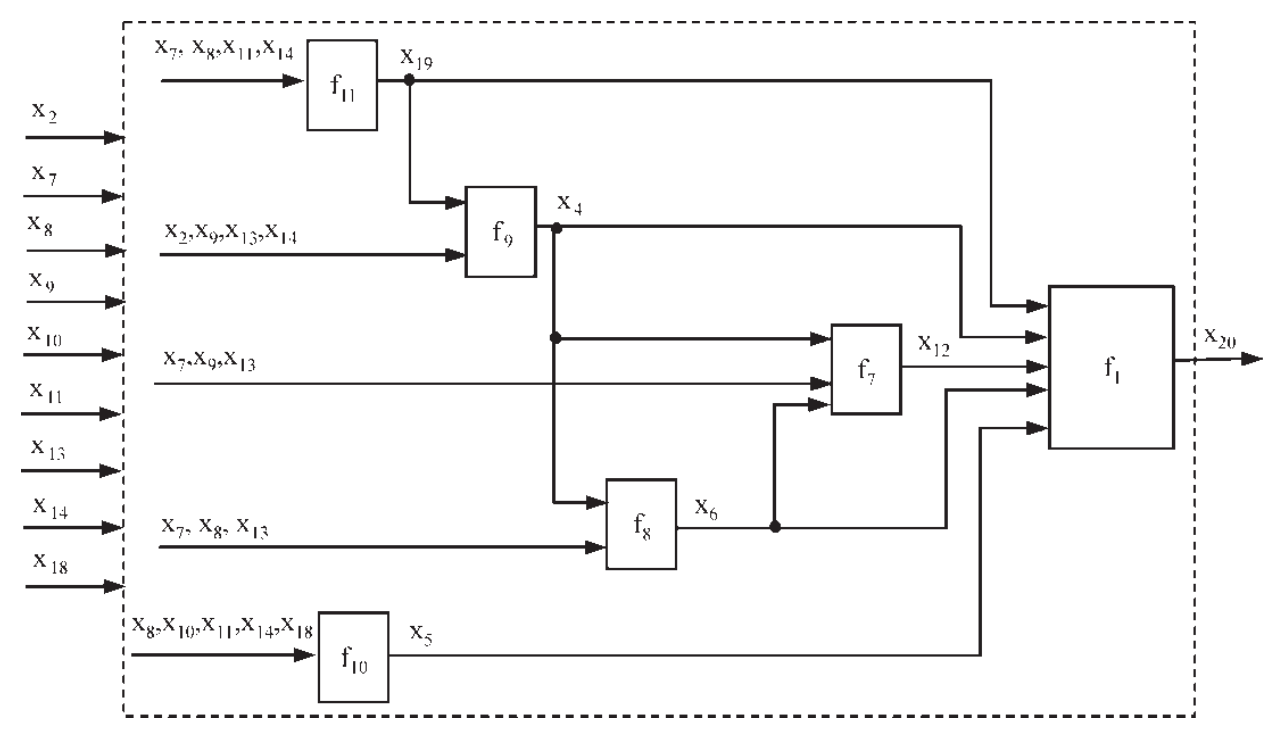

FIGURE 5 Possible structure for the incinerator system found with FIR. 
The structure resulting from the proposed FIR-based algorithm contains 62 binary relations, 39 of which are important, whereas 23 are unimportant.

\section{ASSESSMENT OF RESULTS}

In section 3 a possible structure for the system under study was derived using an FRA-based algorithm as shown in Fig. 3. In section 4, the FIR methodology was used with the same objective (refer to Fig. 5). Up to now results have only been compared on how well those obtained structures encompass the strong binary relations. Both structure systems were derived using 42700 out of 43200 available data points. In this section the last 500 points will be used to validate the output variable from the obtained structures, i.e. models will be assessed with data that were not used to derive them.

First, the topological structure obtained by means of an FRA-based algorithm is used to model the output variable of the incinerator system. FIR models of complexity 5 are found for the $\mathrm{ST}_{6}, \mathrm{ST}_{7}, \mathrm{ST}_{4}, \mathrm{ST}_{2}, \mathrm{ST}_{5}$ and $\mathrm{ST}_{1}$ subsystems so obtaining the behaviour models for the elements of the structure system. In this case, the output variable is modelled from variables $x_{5}, x_{7}, x_{8}$ and $x_{10}$. The FIR optimal model for the $\mathrm{ST}_{1}$ subsystem is given by $X_{20}(t)=f\left\{x_{5}(t-7), X_{8}(t-7), X_{20}(t-3), X_{20}(t-1)\right\}$ with a quality of the model $Q=0.6054$. The real output variable trajectory (depicted by the continuous line) versus the predicted output trajectory (dotted line) are drawn together in Fig. 6. The percent average error for this predicted signal is $10.64 \%$, which is greater than the error reported in Mirats et al. (2002b), which turned to be between 1.25 and $3.64 \%$, depending on the used FIR

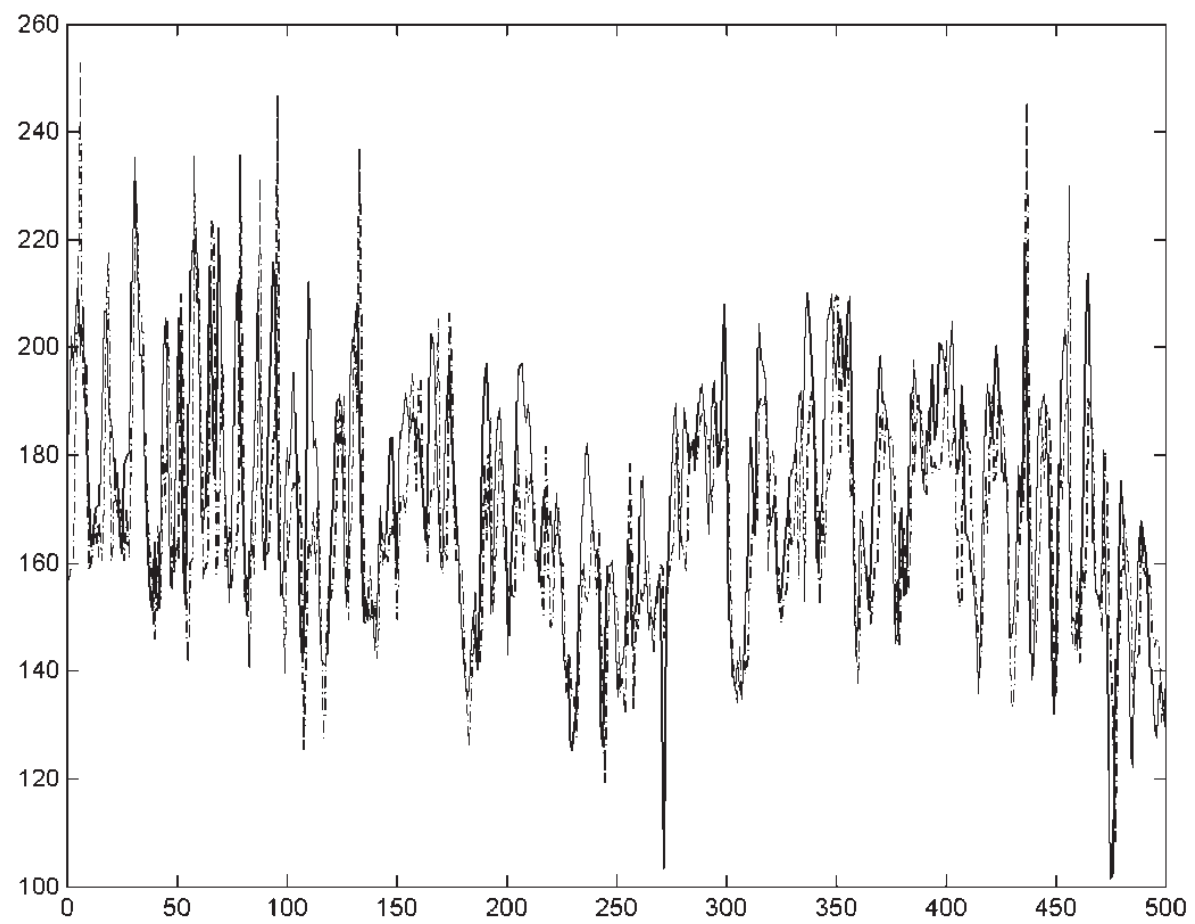

FIGURE 6 Incinerator output variable when using the FRA-obtained structure. Real (continuous line) vs. predicted (dotted line). 


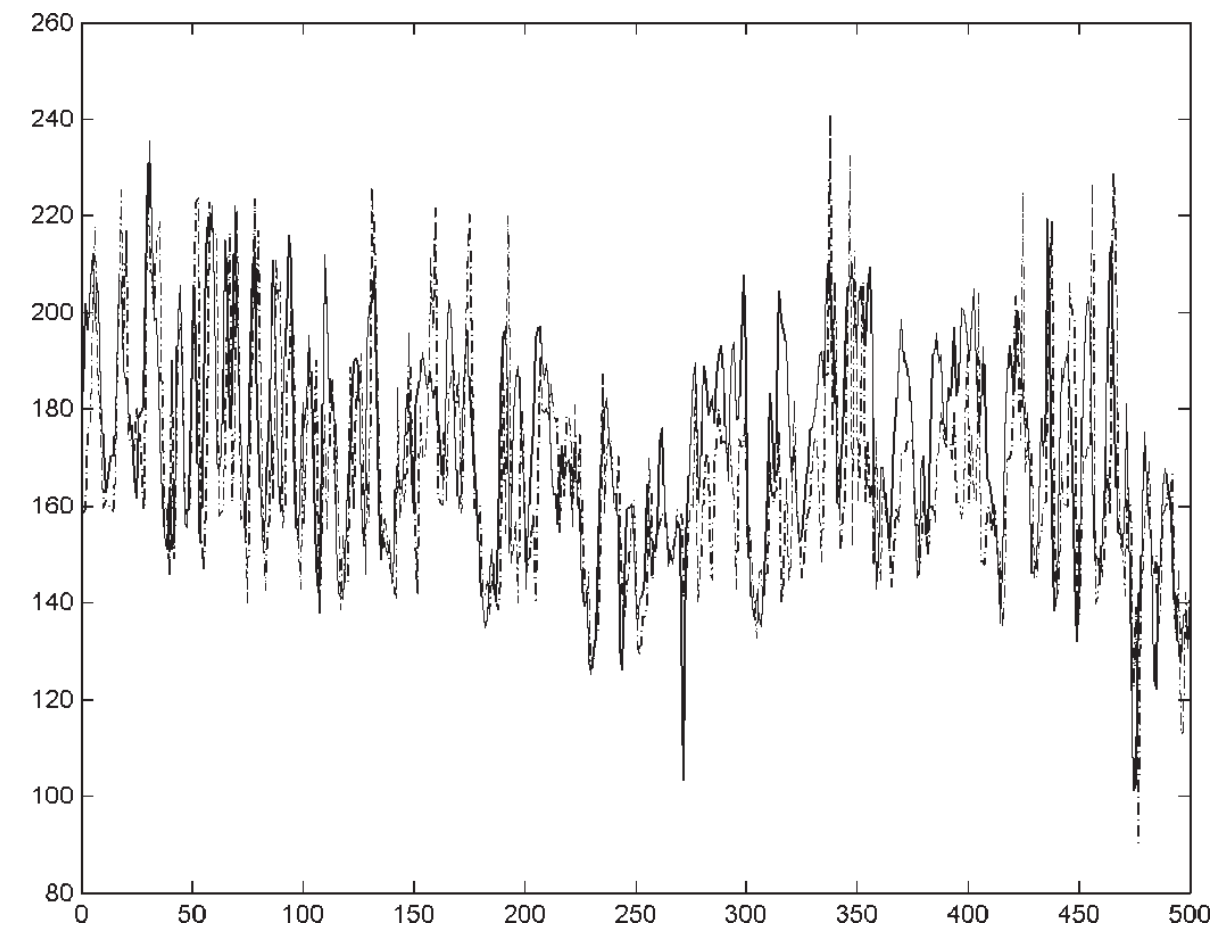

FIGURE 7 Incinerator output variable when using the FIR-obtained structure. Real (continuous line) vs. predicted (dotted line).

model, but still quite acceptable. A structure of a system from its pure data has been obtained, helping to understand the process being analyzed, that pays for the little loss of accuracy when predicting the output variable from the derived structure.

Then, the topological structure obtained by means of the FIR-based algorithm was used to model the output variable of the incinerator system. Again, FIR models of complexity 5 are found for the $f_{11}, f_{10}, f_{9}, f_{8}, f_{7}$ and $f_{1}$ subsystems in Fig. 5. Now, the output variable is modelled from variables $x_{4}, x_{5}, x_{6}, x_{12}$ and $x_{19}$. The FIR optimal model for the $f_{1}$ subsystem is given by the qualitative relation $x_{20}(t)=f\left\{x_{19}(t-7), x_{5}(t-6), x_{20}(t-3), x_{20}(t-1)\right\}$ with a quality of the model $Q=0.6077$. Figure 7 shows the real versus the predicted output trajectory for this particular model. The percent average error for this predicted signal is $9.31 \%$, again greater than the error reported in Mirats et al. (2002b), but slightly less than the error found using the FRA structure.

\section{CONCLUSIONS}

This paper deals with the difficult problem of structure identification in large-scale systems. Two different algorithms are proposed, each of which has the potential of discovering the internal topological structure of a system:

- an algorithm based on fuzzy reconstruction analysis (FRA),

- an algorithm based on FIR.

Let us discuss briefly the pros and cons of these two algorithms.

The FRA-based algorithm is clearly the best from the point of view that FRA, when executed in an exhaustive fashion, looks at every possible projection of every variable onto 
every subspace formed by any subset of the other variables. Unfortunately, the number of variations to be considered grows so rapidly with the number of variables in the system that this approach is at best of academic interest. Only one highly suboptimal FRA-based algorithm has any chance of terminating within a reasonable time span: the single-step refinement algorithm. Unfortunately, even this algorithm becomes highly inefficient for an even modestly large number of variables. Hence FRA-based techniques can only be applied to small- to medium-scale systems.

FRA-based algorithms operate on the binary structure of the system. Whereas the composite structure can be derived from the binary structure, it is not possible to derive the topological structure (which is our aim) from the composite structure. A heuristic algorithm was proposed that derives a topological structure directly from the relative strengths of the binary relations found by the single-step refinement algorithm of FRA. The algorithm has two major drawbacks.

1. The number of inputs of each subsystem was limited to four. Why four? Why not three of five? Shouldn't the number of possible inputs depend on the quantity and quality of available observational data? FIR clearly does a better job at selecting inputs in a dynamical and rational fashion.

2. The algorithm only considers the strengths of binary relations between the individual inputs and the output of each subsystem. The relative strengths of the binary relations among the inputs are not considered. Hence FRA chooses those variables with the largest strengths of binary relations to the output, but maybe two of those input variables have strong binary relations between them so that another input variable might offer more additional information in spite of exhibiting a smaller strength of its binary relation with the output.

The FIR-based algorithm makes use of all the variables to select an optimal mask for the output variable. It then works its way back towards the inputs, treating each of the inputs of this submodel in turn as an internal variable, proposing an FIR-model for it. The algorithm continues until all variables are accounted for. The FIR-based approach has the advantage that it makes use of a methodology that was designed as a tool for the generation of simulation models with optimal prediction power. Hence the resulting FIR-model decomposition should offer excellent prediction capabilities. The FIR-based algorithm also has its drawbacks.

1. The algorithm, as proposed, considers the weaker inputs first as internal variables. This makes sense, because it allows sensors to be reserved for the stronger variables. Yet in the given example, all inputs of the last stage turned out to be made into internal variables. Wouldn't it then have made more sense to model the stronger inputs first? Doesn't FIR try to make a compromise in each model between completeness of available information (many inputs) and complexity of the model (few inputs), in order to optimise the usage of the available observational data? Isn't there a "conflict of interest" between choosing stronger versus weaker variables first?

2. The main goal of this research effort is to find techniques that reduce the workload for FIR by reducing the number of potential input variables to be considered. Yet already in the first step of this algorithm, a complete FIR problem is being solved, i.e. the main purpose of the investigation is defeated. Clearly, FIR is not suitable as a tool for dealing with large-scale systems directly.

It may be interesting to compare the two different approaches from the point of view of their coverage of the important binary relations. To this end, the binary relations of the incinerator 
system were reordered in terms of decreasing relative strengths, and next to each binary relation it was marked whether or not it was captured by any or all of the two proposed algorithms in Table IV.

Both algorithms do a good job at capturing the majority of the important binary relations. Let us look at the most important binary relation relating variables $X_{12}$ and $X_{14}$ with each other.

TABLE IV Capturing of binary relations by the three proposed structure identification algorithms

\begin{tabular}{|c|c|c|c|}
\hline Importance of binary relation & Binary relation & FRA-based method & FIR-based method \\
\hline 0.031104 & 12,14 & $\sqrt{ }$ & $\square$ \\
\hline 0.031102 & 6,19 & $\sqrt{ }$ & $\sqrt{ }$ \\
\hline 0.028183 & 4,6 & $\square$ & $\sqrt{ }$ \\
\hline 0.027111 & 2,13 & $\sqrt{ }$ & $\sqrt{ }$ \\
\hline 0.025047 & 4,12 & $\square$ & $\sqrt{ }$ \\
\hline 0.024819 & 12,13 & $\sqrt{ }$ & $\sqrt{ }$ \\
\hline 0.024196 & 6,7 & $\sqrt{ }$ & $\sqrt{ }$ \\
\hline 0.023871 & 8,9 & $\sqrt{ }$ & $\square$ \\
\hline 0.023185 & 6,12 & $\sqrt{ }$ & $\sqrt{ }$ \\
\hline 0.023164 & 13,14 & $\sqrt{ }$ & $\sqrt{ }$ \\
\hline 0.023159 & 10,18 & $\sqrt{ }$ & $\sqrt{ }$ \\
\hline 0.023143 & 8,14 & $\sqrt{ }$ & $\sqrt{ }$ \\
\hline 0.022214 & 7,12 & $\sqrt{ }$ & $\sqrt{ }$ \\
\hline 0.022142 & 6,8 & $\sqrt{ }$ & $\sqrt{ }$ \\
\hline 0.021783 & 10,20 & $\sqrt{ }$ & $\square$ \\
\hline 0.021566 & 7,8 & $\sqrt{ }$ & $\sqrt{ }$ \\
\hline 0.021411 & 5,11 & $\sqrt{ }$ & $\sqrt{ }$ \\
\hline 0.021365 & 7,19 & $\sqrt{ }$ & $\sqrt{ }$ \\
\hline 0.021305 & 14,18 & $\square$ & $\sqrt{ }$ \\
\hline 0.021164 & 11,14 & $\sqrt{ }$ & $\sqrt{ }$ \\
\hline 0.021082 & 2,11 & $\sqrt{ }$ & $\square$ \\
\hline 0.020031 & 7,14 & $\sqrt{ }$ & $\sqrt{ }$ \\
\hline 0.019011 & 11,13 & $\sqrt{ }$ & $\square$ \\
\hline 0.018401 & 9,13 & $\square$ & $\sqrt{ }$ \\
\hline 0.018399 & 8,10 & $\sqrt{ }$ & $\sqrt{ }$ \\
\hline 0.018324 & 8,19 & $\square$ & $\sqrt{ }$ \\
\hline 0.018102 & 8,20 & $\sqrt{ }$ & $\square$ \\
\hline 0.018031 & 10,13 & $\sqrt{ }$ & $\square$ \\
\hline 0.016835 & 4,19 & $\square$ & $\sqrt{ }$ \\
\hline 0.016339 & 7,13 & $\square$ & $\sqrt{ }$ \\
\hline 0.016134 & 4,10 & $\sqrt{ }$ & $\square$ \\
\hline 0.016028 & 2,12 & $\sqrt{ }$ & $\square$ \\
\hline 0.015811 & 9,12 & $\square$ & $\sqrt{ }$ \\
\hline 0.015678 & 10,12 & $\square$ & $\square$ \\
\hline 0.015141 & 5,14 & $\sqrt{ }$ & $\sqrt{ }$ \\
\hline 0.015137 & 6,9 & $\sqrt{ }$ & $\sqrt{ }$ \\
\hline 0.014957 & 11,12 & $\sqrt{ }$ & $\square$ \\
\hline 0.014753 & 9,14 & $\sqrt{ }$ & $\sqrt{ }$ \\
\hline 0.014634 & 9,11 & $\square$ & $\square$ \\
\hline 0.014601 & 6,13 & $\square$ & $\sqrt{ }$ \\
\hline 0.014261 & 4,11 & $\square$ & $\square$ \\
\hline 0.014242 & 7,20 & $\sqrt{ }$ & $\square$ \\
\hline 0.014203 & 2,9 & $\square$ & $\sqrt{ }$ \\
\hline 0.013597 & 10,14 & $\square$ & $\sqrt{ }$ \\
\hline 0.013272 & 5,8 & $\sqrt{ }$ & $\sqrt{ }$ \\
\hline 0.013191 & 7,18 & $\square$ & $\square$ \\
\hline 0.013151 & 4,8 & $\sqrt{ }$ & $\sqrt{ }$ \\
\hline 0.013029 & 4,13 & $\sqrt{ }$ & $\sqrt{ }$ \\
\hline 0.012323 & 7,9 & $\sqrt{ }$ & $\sqrt{ }$ \\
\hline 0.012272 & 4,9 & $\square$ & $\sqrt{ }$ \\
\hline 0.012103 & 4,7 & $\square$ & $\sqrt{ }$ \\
\hline 0.011106 & 5,20 & $\sqrt{ }$ & $\sqrt{ }$ \\
\hline 0.011067 & 12,19 & $\sqrt{ }$ & $\sqrt{ }$ \\
\hline 0.01105 & 2,8 & $\square$ & $\square$ \\
\hline 0.010561 & 2,14 & $\sqrt{ }$ & $\sqrt{ }$ \\
\hline 0.009398 & 2,20 & $\square$ & $\square$ \\
\hline
\end{tabular}


TABLE IV - continued

\begin{tabular}{|c|c|c|c|}
\hline Importance of binary relation & Binary relation & FRA-based method & FIR-based method \\
\hline 0.008869 & 11,18 & $\square$ & $\sqrt{ }$ \\
\hline 0.008572 & 4,14 & $\square$ & $\sqrt{ }$ \\
\hline 0.008524 & 2,18 & $\square$ & $\square$ \\
\hline 0.008464 & 10,11 & $\square$ & $\sqrt{ }$ \\
\hline 0.008357 & 11,19 & $\square$ & $\sqrt{ }$ \\
\hline 0.008176 & 11,20 & $\square$ & $\square$ \\
\hline 0.006091 & 4,18 & $\sqrt{ }$ & $\square$ \\
\hline 0.006032 & 8,13 & $\sqrt{ }$ & $\sqrt{ }$ \\
\hline 0.005103 & 4,20 & $\square$ & $\sqrt{ }$ \\
\hline 0.004083 & 5,18 & $\square$ & $\sqrt{ }$ \\
\hline 0.004012 & 18,20 & $\square$ & $\square$ \\
\hline 0.002862 & 18,19 & $\square$ & $\square$ \\
\hline 0.002243 & 19,20 & $\square$ & $\sqrt{ }$ \\
\hline 0.000661 & 5,9 & $\square$ & $\square$ \\
\hline 0.000367 & 7,10 & $\sqrt{ }$ & $\square$ \\
\hline 0.000297 & 9,18 & $\square$ & $\square$ \\
\hline 0.000286 & 2,19 & $\square$ & $\sqrt{ }$ \\
\hline 0.000282 & 8,18 & $\sqrt{ }$ & $\sqrt{ }$ \\
\hline 0.000258 & 12,20 & $\square$ & $\sqrt{ }$ \\
\hline 0.000221 & 7,11 & $\square$ & $\sqrt{ }$ \\
\hline 0.000216 & 9,20 & $\square$ & $\square$ \\
\hline 0.000214 & 9,19 & $\square$ & $\sqrt{ }$ \\
\hline 0.000174 & 6,20 & $\square$ & $\sqrt{ }$ \\
\hline 0.000157 & 10,19 & $\square$ & $\square$ \\
\hline 0.000135 & 13,20 & $\square$ & $\square$ \\
\hline 0.000121 & 2,4 & $\square$ & $\sqrt{ }$ \\
\hline 0.000116 & 12,18 & $\square$ & $\square$ \\
\hline 0.000115 & 8,12 & $\square$ & $\square$ \\
\hline 0.000109 & 14,19 & $\square$ & $\sqrt{ }$ \\
\hline 0.000103 & 13,18 & $\sqrt{ }$ & $\square$ \\
\hline 0.000102 & 8,11 & $\sqrt{ }$ & $\sqrt{ }$ \\
\hline 0.000099 & 14,20 & $\square$ & $\square$ \\
\hline 0.000084 & 6,14 & $\sqrt{ }$ & $\square$ \\
\hline 0.000079 & 6,18 & $\square$ & $\square$ \\
\hline 0.000075 & 5,19 & $\square$ & $\sqrt{ }$ \\
\hline 0.000067 & 5,6 & $\square$ & $\sqrt{ }$ \\
\hline 0.000067 & 9,10 & $\square$ & $\square$ \\
\hline 0.000042 & 2,10 & $\square$ & $\square$ \\
\hline 0.000037 & 13,19 & $\square$ & $\sqrt{ }$ \\
\hline 0.000033 & 4,5 & $\square$ & $\sqrt{ }$ \\
\hline 0.000031 & 2,5 & $\square$ & $\square$ \\
\hline 0.000028 & 6,10 & $\square$ & $\square$ \\
\hline 0.000026 & 5,7 & $\sqrt{ }$ & $\square$ \\
\hline 0.000024 & 2,6 & $\square$ & $\square$ \\
\hline 0.000019 & 5,12 & $\square$ & $\sqrt{ }$ \\
\hline 0.000017 & 5,10 & $\sqrt{ }$ & $\sqrt{ }$ \\
\hline 0.000011 & 2,7 & $\square$ & $\square$ \\
\hline 0.000011 & 5,13 & $\square$ & $\square$ \\
\hline 0.000001 & 6,11 & $\square$ & $\square$ \\
\hline
\end{tabular}

This binary relation is captured by the FRA-based algorithm only. The way both algorithms operate, by working their way back from the output to the inputs, a strong binary relation between inputs may be missed if neither of these inputs exhibits a strong binary relation with the output, as is the case in the given example. Yet, and this is interesting indeed, the FIR-based algorithm made use of $X_{12}$ as an internal variable, but did not include $X_{14}$ among the inputs to be used by the optimal mask. Evidently, it found that $X_{12}$ is better predictable from a combination of other variables. The FIR-based approach also employs a variety of less important variables as part of its inputs, because they still contain useful information that can be exploited. 
The reader is reminded that FIR is truly a simulation tool, whereas FRA and the statistical technique are structuring tools. It is very promising to recognize that techniques as different as FRA and the correlation method indeed come up with a relatively similar set of important binary relations.

\section{References}

de Albornoz, A. (1996) "Inductive reasoning and reconstruction analysis: two complementary tools for qualitative fault monitoring of large-scale systems", Ph.D. thesis (Department Llenguatges i sistemes informàtics, Universitat Politècnica de Catalunya).

Ashby, W.R. (1965) "Measuring the internal information exchange in a system", Cybernetica 8(1), 5-22.

Broekstra, G. (1976) "Constraint analysis and structure identification", Annals of Systems Research I(5), 67-80.

Broekstra, G. (1977) "Constraint analysis and structure identification”, Annals of Systems Research II(6), 1-20.

Broekstra, G. (1978) "On the representation and identification of structure systems", International Journal of Systems Science 9(11), 1271-1293.

Cavallo, R.E. and Klir, G.J. (1979a) "Reconstructability analysis of multi-dimensional relations: a theoretical basis for computer-aided determination of acceptable systems models", International Journal of General Systems $\mathbf{5}(3), 143-171$.

Cavallo, R.E. and Klir, G.J. (1979b) "The structure of reconstructable relations: a comprehensive study", Journal of Cybernetics 9(4), 399-413.

Cavallo, R.E. and Klir, G.J. (1981a) "Reconstructability analysis: overview and bibliography”, International Journal of General Systems 7(1), 1-6.

Cavallo, R.E. and Klir, G.J. (1981b) "Reconstructability analysis: evaluation of reconstruction hypotheses", International Journal of General Systems 7(1), 7-32.

Cellier, F.E. (1991) Continuous System Modelling (Springer-Verlag, New York).

Conant, R.C. (1972) "Detecting subsystems of a complex system", IEEE Trans. Syst. Man Cybernet. SMC 2(4), $550-553$.

Conant, R.C. (1976) "Laws of information which govern systems", IEEE Trans. Syst Man Cybernet. SMC 6(4), $240-255$.

Gaines, B.R. (1977) "System identification, approximation and complexity", International Journal of General Systems 3(3), 145-174.

Kengerlinsky, G.A. (1978) "An informational approach to the decomposition of complex systems", Engineering Cybernetics 16(1), 91-97.

Klir, G.J. and Uyttenhove, H.J.J. (1979) "Procedures for generating reconstruction hypotheses in the reconstructability analysis", International Journal of General Systems 5, 231-246.

Klir, G.J. (1981) "On systems methodology and inductive reasoning: the issue of parts and wholes", General Systems Yearbook 26, 29-38.

Klir, G.J. (1985) Architecture of System Problem Solving (Plenum Press, New York).

Klir, G.J. (1991) "Aspects of uncertainty in qualitative systems modelling", In: Fishwick and Luker, eds, Qualitative Simulation Modelling and Analysis (Springer-Verlag, New York).

Krippendorff, K. (1981) "An algorithm for identifying structural models of multi-variate data", International Journal of General Systems 7(1), 63-79.

Krippendorff, K. (1986) Information Theory: Structural Models for Qualitative Data (Quantitative Applications in the Social Sciences 62), (Sage, Beverly Hills).

Madden, R.F. and Ashby, W.R. (1972) "The identification of many-dimensional relations", International Journal of Systems Science 3(4), 343-356.

Mirats, J.M. and Verde, R. (2000) "Subsystem identification within a complex system", Simulation and modelling: enablers for a better quality of life, $14^{\text {th }}$ European simulation multiconference (Ghent, Belgium) May 23-26, pp $773-777$.

Mirats, J.M., Cellier, F.E., Huber, R.H. and Qin, S.J. (2002a) "On the selection of variables for qualitative modelling of dynamical systems", International Journal of General Systems 31(5), 435-467.

Mirats, J.M., Cellier, F.E. and Huber, R.H. (2002b) "Variable selection procedures and efficient suboptimal mask search algorithms in fuzzy inductive reasoning", International Journal of General Systems 31(5), 469-498.

Mirats, J.M. (2002) "Qualitative modelling of complex systems by means of fuzzy inductive reasoning. Variable selection and search space reduction", Ph.D. dissertation, Institut de Robòtica i Informàtica Industrial, Universitat Politècnica de Catalunya (Barcelona, Spain).

Uyttenhove, H.J. (1978) "Computer aided systems modelling: an assemblage of methodological tools for systems problem solving", Ph.D. dissertation, School of advanced technology, University of New York (SUNY-Binghamton, USA).

Valle, S., Weihua Li and Qin, S.J. (1999) "Selection of the number of principal components: the variance of the reconstruction error criterion with a comparison to other methods", Industrial Engineering Chemical Research 38, 4389-4401. 


\section{APPENDIX}

The process analysed in this work is a thermal incinerator. The description given here is a general description of the functioning of these kinds of systems (Valle et al., 1999). In this unit, high temperature and chemical reactions burn up process fumes, and change them into harmless carbon dioxide and water vapours, which are then released through a stack into the atmosphere. The fumes processed in the incinerator are commonly called $\mathrm{NO}_{\mathrm{x}}$ fumes, and mainly consist of nitric oxide (NO), nitrogen dioxide $\left(\mathrm{NO}_{2}\right)$, and nitrous oxide $\left(\mathrm{N}_{2} \mathrm{O}\right)$. Hydrogen cyanide (HCN) is also present. The gases are converted into nitrogen and water vapour in a three-stage combustion process. The first stage is reduction, the second is re-oxidation, and the third is catalytic oxidation. A de-mineralised water (DMW) heat exchanger is located between the second and third combustion stages (Fig. A1).

Data were gathered up from the system at a sampling rate of $1 \mathrm{~min}$ and 43200 data points were recorded. The considered output variable for this study is the emission of $\mathrm{NO}_{\mathrm{x}}$ gas. Table A1 gives a short description of the variables in the system that this paper deals with. Figure A1 shows a general diagram describing the layout of the system.

The reduction section is a large natural gas (methane) furnace. The air for the combustion is provided by the vent header (DNT), or stripper vent header (DNA). The burning of natural gas provides all the heat required for the reduction stage. There are four major chemical reactions that occur in the reduction section furnace.

1. Burning of natural gas $\mathrm{CH}_{4}+2 \mathrm{O}_{2} \Rightarrow 2 \mathrm{H}_{2} \mathrm{O}+\mathrm{CO}_{2}$

2. $\mathrm{NO}_{\mathrm{x}}$ gas is destroyed by $\mathrm{CH}_{4}+2 \mathrm{NO}_{2} \Rightarrow \mathrm{N}_{2}+2 \mathrm{H}_{2} \mathrm{O}+\mathrm{CO}_{2}$

3. Additional fuel is added without air to increase the reaction between natural gas and the $\mathrm{NO}_{\mathrm{x}}$, and to remove any trace of oxygen in the system $\mathrm{CH}_{4}+4 \mathrm{NO} \Rightarrow 2 \mathrm{~N}_{2}$ $+2 \mathrm{H}_{2} \mathrm{O}+\mathrm{CO}_{2}$

4. $\mathrm{CO}_{2}$ gas is destroyed by $\mathrm{CH}_{4}+\mathrm{CO}_{2} \Rightarrow 2 \mathrm{CO}+2 \mathrm{H}_{2}$.

The hot gases leaving the reduction furnace thus consist of carbon monoxide, nitrogen, hydrogen, water vapour, carbon dioxide, $\mathrm{NO}_{\mathrm{x}}$ (below $200 \mathrm{ppm}$ ), and $\mathrm{HCN}$

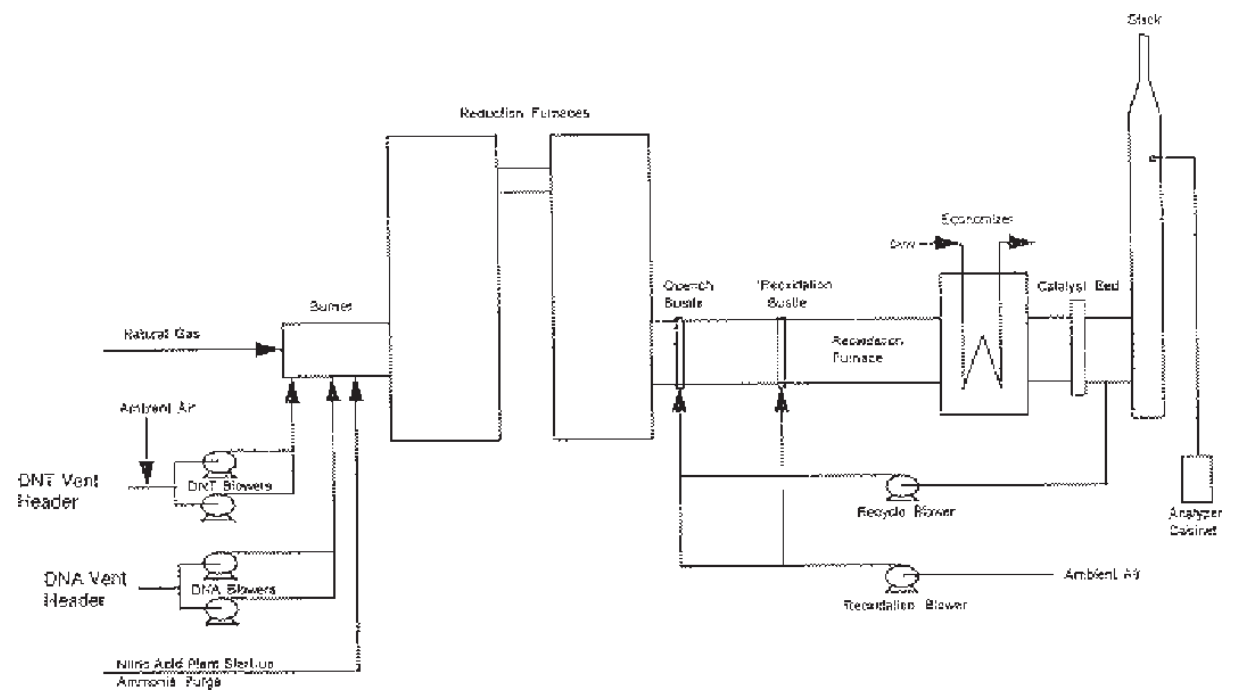

FIGURE A1 Incineration process scheme. 
TABLE A1 Variable description in the garbage incinerator plant

\begin{tabular}{llll}
\hline Variable & \multicolumn{1}{c}{ Physical meaning } & Variable & \multicolumn{1}{c}{ Physical meaning } \\
\hline 1 (input) & DNT unit one vent (SCFM) & 11 (input) & Column B top pressure (inch W) \\
2 (input) & DNT unit two vent (SCFM) & 12 (input) & Column C top pressure (inch W) \\
3 (input) & A column overhead (F) & 13 (input) & Column D top pressure (inch W) \\
4 (input) & C column overhead (F) & 14 (input) & DP on A column (PSIG) \\
5 (input) & DNT vent rate (SCFM) & 15 (input) & DP on B column (PSIG) \\
6 (input) & Stack gas recycle (ACFM) & 16 (input) & DP on C column (PSIG) \\
7 (input) & Stack gas recycle (F) & 17 (input) & DP on D column (PSIG) \\
8 (input) & Strip vent rate (SCFM) & 18 (input) & Excess O2 in stack (\%) \\
9 (input) & DNT vent head VA (WC) & 19 (input) & Reduction furnace (F) \\
10 (input) & Column A top pressure (inch W) & 20 (output) & NOx, (PPM) \\
\hline
\end{tabular}

(below $500 \mathrm{ppm}$ ). After the reduction furnace hot gases are quenched by mixing them with cooler recycled stack gas. The quenched gas then flows into the stage section called the re-oxidation stage. This section is not used for $\mathrm{NO}_{\mathrm{x}}$ abatement, but to convert $\mathrm{CO}$ and $\mathrm{H}_{2}$, the combustibles from the reduction furnace, into $\mathrm{CO}_{2}$ and water vapour. In this section fuel gas is not used, and a blower ads ambient air to the section. Two additional reactions occur:

$$
2 \mathrm{CO}+2 \mathrm{O}_{2} \Rightarrow \mathrm{O}_{2}+2 \mathrm{CO}_{2} \quad 2 \mathrm{H}_{2}+\mathrm{O}_{2} \Rightarrow 2 \mathrm{H}_{2} \mathrm{O} .
$$

The hot gases from the re-oxidation section then flow through an economiser where they are cooled by de-mineralising water. The hot DMW is then routed to the plant. Gases exiting the economiser flow through a honeycomb grid of platinum catalyst, where the $\mathrm{CO}$ and the organics are converted to inert flue gases before their discharge from the stack to the atmosphere:

$$
\mathrm{CO}+2 \mathrm{HCN}+\mathrm{C}_{6} \mathrm{H}_{5} \mathrm{CH}_{3}+\mathrm{O}_{2} \Rightarrow \mathrm{CO}_{2}+\mathrm{H}_{2} \mathrm{O}+\mathrm{N}_{2} .
$$

Analyser probes in the stack monitor the oxygen, $\mathrm{CO}, \mathrm{CO}_{2}$, and $\mathrm{NO}_{\mathrm{x}}$ levels.

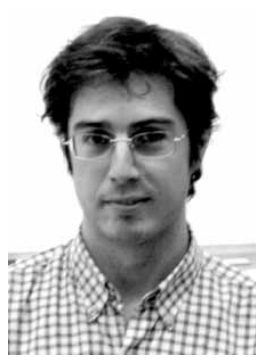

Josep M. Mirats Tur graduated as a Enginyer Superior en Telecomunications in 1995.at the Universitat Politècnica de Catalunya (UPC). During his university stage he was on the board of directors of the Fòrum de Telecomunicacions Association during 1993 and 1994, a student member of the IEEE, and a founding partner of the IEEE student chapter, Aerospace and Electronics System Society (AESS), in Catalonia. In 1994, he was one of the founders of the Annual Robot Competition created by AESS. In 2000, he obtained his Ph.D. degree at the Institut of Robotics in Barcelona. In 2002, he was awarded with the mention of European Doctor while working for the Consell Insular d'Eivissa i Formentera (Government of Eivissa in the Balearic Islands) in the implementation of a new telecommunications systems for voice, fixed and mobile, and data between their different buildings. Nowadays he holds a Post-doctoral position at the Institute of Robotics in Barcelona. His main areas of interest are model simplification, fuzzy inductive reasoning, reconstruction analysis, extracting knowledge from environment, field robotics, cooperating robots, knowledge integration, and sensor data fusion. 


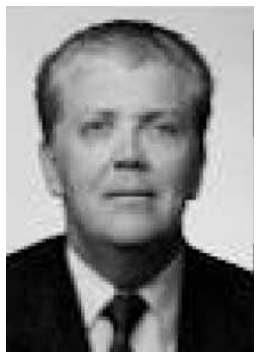

Rafael M. Huber received his Ingeniero Industral (Electrical Engineering branch) and his Ph.D. in Ingeniería Industrial in 1976, both from the Universitat Politècnica de Catalunya (UPC). His present position is Catedrático de Universidad (Professor) at the Automatic Control Department of the UPC and nowadays he is serving as director of the Instituto de Robótica e Informática Industrial (IRI) depending of the UPC and the Spanish Council of Scientific Research (CSIC). His main scientific interests concern modelling and simulation methodology and the design of advanced simulation environments. His present research focuses on qualitative modelling and simulation and its application to dynamic systems fault detection and diagnosis. He has been involved as research engineer or research head in projects with the Spanish industry, the Comisión Interministerial de Ciencia y Tecnología (CICYT), the CSIC, the European Space Agency and the U.S. National Science Foundation. Prof. Huber has authored or co-authored more than forty technical publications and edited two books related to continuous system modelling.

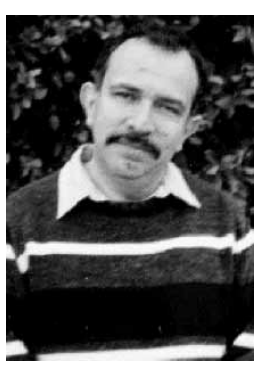

François E. Cellier received his B.S. degree in Electrical Engineering from the Swiss Federal Institute of Technology (ETH) Zürich in 1972, his M.S. degree in Automatic Control in 1973, and his Ph.D. degree in Technical Sciences in 1979, all from the same university. Dr Cellier joined the University of Arizona in 1984 as Associate Professor. His main scientific interests concern modelling and simulation methodologies, and the design of advanced software systems for simulation, computer-aided modelling, and computer-aided design. Dr Cellier has authored or co-authored more than eighty technical publications, and he has edited four books. He recently published his first textbook on Continuous System Modelling (SpringerVerlag New York, 1991). He served as General Chairman or Program Chairman of many international conferences, most recently ICBGM'93 (SCS International Conference on Bond Graph Modeling, San Diego, January 1993), CACSD'94 (IEEE/IFAC Symposium on Computer-Aided Control System Design, Tucson, March 1994), ICQFN'94 (SCS International Conference on Qualitative Information, Fuzzy Techniques, and Neural Networks in Simulation, Barcelona, June 1994), ICBGM'95 (Las Vegas, January 1995), WMC'96 (SCS Western Simulation MultiConference, San Diego, January 1996), WMC'97 (Tucson, January 1997). He is Associate Editor of several simulation-related journals, and he served as vice-chairman on two committees for standardization of simulation and modelling software. Dr Cellier was promoted to the rank of Full Professor in 1997. 